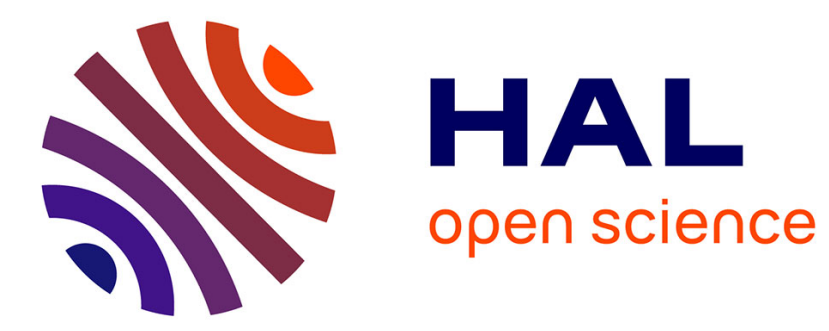

\title{
Masers solides à pompage optique
}

\author{
J.-P. Gayda, J. Hervé
}

\section{To cite this version:}

J.-P. Gayda, J. Hervé. Masers solides à pompage optique. Revue de Physique Appliquée, 1971, 6 (2), pp.177-179. 10.1051/rphysap:0197100602017700 . jpa-00243518

\section{HAL Id: jpa-00243518 https://hal.science/jpa-00243518}

Submitted on 1 Jan 1971

HAL is a multi-disciplinary open access archive for the deposit and dissemination of scientific research documents, whether they are published or not. The documents may come from teaching and research institutions in France or abroad, or from public or private research centers.
L'archive ouverte pluridisciplinaire HAL, est destinée au dépôt et à la diffusion de documents scientifiques de niveau recherche, publiés ou non, émanant des établissements d'enseignement et de recherche français ou étrangers, des laboratoires publics ou privés. 


\title{
MASERS SOLIDES A POMPAGE OPTIQUE
}

\author{
par J.-P. GAYDA et J. HERVÉ \\ Laboratoire d'Electronique, Université de Provence, Saint-Jérôme, 13, Marseille (13e)
}

\begin{abstract}
Résumé. - Analyse du fonctionnement des masers pompés sélectivement par laser (mise en évidence d'une pulsation signal maximale $\omega_{0}$ pour le régime stationnaire et d'un effet maser transitoire pour $\omega_{s}>\omega_{0}$ ) et des masers utilisant un pompage dans une bande large avec effet de dichroïsme (condition assez sévère sur le temps de relaxation de l'état fondamental). - Etude du bruit d'émission spontanée et de la possibilité pour la température de bruit d'être inférieure à celle de l'échantillon. - Description des réalisations : masers pulsés à rubis pompés par laser (performances actuelles : $\left.77^{\circ} \mathrm{K}, 50 \mathrm{GHz}\right)$ et masers continus à ions $\mathrm{Tm}^{++}$pompés par dichroïsme $\left(4^{\circ} \mathrm{K}, 18 \mathrm{GHz}\right)$.

Abstract. - Analysis of working conditions in laser pumped masers (existence of a maximum signal frequency $\omega_{0}$ for steady working and of a transient maser effect when $\omega_{s}>\omega_{0}$ ) and in masers using the pumping into a broad band with dichroism properties (severe conditions upon ground state relaxation times). - Study of the spontaneous emission noise and of the feasibility of a noise temperature much smaller than the sample one. - Description of realizations : pulsed laser pumped ruby masers (present performances : $77^{\circ} \mathrm{K}, 50 \mathrm{GHz}$ ) and continuous $\mathrm{Tm}^{++}$masers pumped by dichroism $\left(4^{\circ} \mathrm{K}, 18 \mathrm{GHz}\right)$.
\end{abstract}

Introduction. - Les possibilités de pompage optique dans les solides ont été étudiées à partir de 1958 [13]. Plusieurs auteurs ont proposé de réaliser des masers utilisant comme pompage, soit la saturation sélective d'une transition étroite au moyen d'un laser [4-6], soit un effet de dichroïsme circulaire dans une bande d'absorption large [7]. On prévoyait que de tels dispositifs auraient, sur les masers à pompage hyperfréquence, les avantages suivants:

a) L'inversion de population peut se produire jusqu'à des températures plus élevées.

b) La température de bruit peut être notablement inférieure à celle de l'échantillon.

c) La fréquence de travail n'est pas limitée par l'absence de source de pompage efficace.

Nous étudions ici les conditions de fonctionnement et examinons dans quelle mesure les réalisations pratiques [8-15] ont confirmé les prévisions. Nous appellerons conditions optimales, celles pour lesquelles l'inversion de population devient indépendante de la puissance de pompage.

1. Principe du maser pompé par laser. - Discutons l'effet maser au moyen du modèle simplifié suivant [6] :

a) L'état macroscopique est spécifié par des populations $n_{\mathbf{i}}$.

b) Les populations des trois états impliquées (Fig. 1) sont isolées.

c) Le pompage affecte sélectivement le couple de niveaux 1-3 avec la probabilité de transition $W_{\mathrm{p}}$.

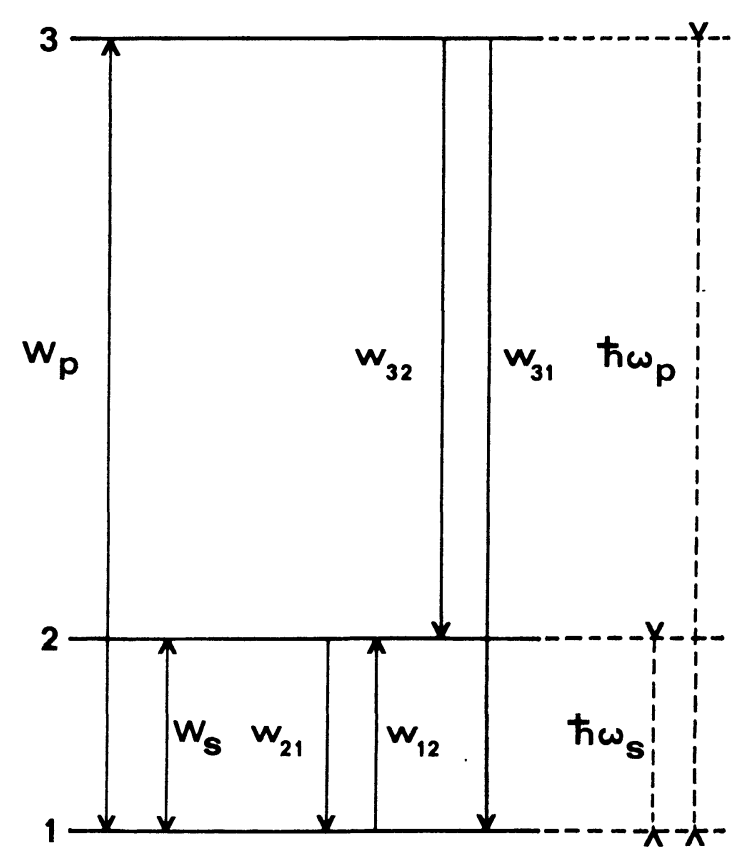

Fig. 1. - Transitions importantes dans le maser à pompage sélectif par laser.

d) La probabilité de transition $W_{\mathrm{s}}$ due au champ «signal » est indépendante de l'état du système (maser amplificateur à faible niveau).

e) Les probabilités de transition intervenant pour la restauration de l'équilibre thermique sont les $w_{i j}$ de de la figure 1.

f) $\hbar \omega_{\mathrm{s}} \ll k T ; \hbar \omega_{\mathrm{p}} \gg k T$. 
Alors la solution stationnaire s'écrit :

$$
\begin{aligned}
& \left(\frac{n_{2}-n_{1}}{n_{1}}\right)_{\mathrm{st}}= \\
& =\frac{w_{21}}{W_{\mathrm{s}}+w_{21}}\left(\frac{w_{32}}{w_{21}} \frac{W_{\mathrm{p}}}{W_{\mathrm{p}}+w_{31}+w_{32}}-\frac{\hbar \omega_{\mathrm{s}}}{k T}\right) .
\end{aligned}
$$

On voit que l'inversion se produit pour les $\omega_{\mathrm{s}}$ supérieures à une certaine borne $\omega_{0}$.

Les conditions optimales se traduisent par:

$$
W_{\mathrm{p}} \gg\left(w_{31}+w_{32}\right)
$$

et, dans ce cas, $\omega_{0}=(k T / \hbar) w_{32} w_{21}^{-1}$.

Lorsque $\omega_{\mathrm{S}} \ll \omega_{0}$, l'inversion de population ne dépend de $T$ que par l'intermédiaire de $w_{32}$ et $w_{21}$ et peut rester notable à température assez élevée. Dans le cas du maser à pompage hyperfréquence, l'expression analogue à (1) comporte un facteur de Boltzmann [16-17] qui impose, en pratique, une basse température de fonctionnement.

Lorsque $\omega_{\mathrm{s}}>\omega_{0}$, on peut observer un effet maser transitoire [9], [12] : si le pompage est assez puissant, $n_{1}$ et $n_{3}$ s'égalisent en un temps très court par rapport à $w_{21}^{-1}$ et $w_{12}^{-1}$; l'effet maser s'établit alors et dure jusqu'à ce que la relaxation ait réarrangé les populations. La figure 2 représente la solution des équations d'évolution.

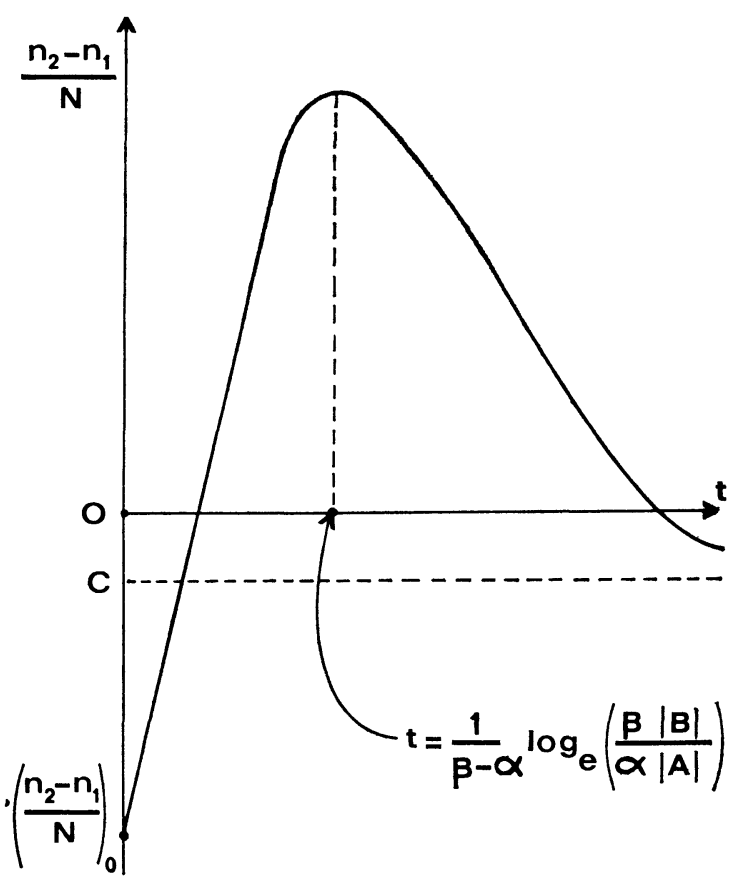

FIG. 2. - Régime transitoire $: \frac{n_{2}-n_{1}}{N}=A \mathrm{e}^{-\alpha t}+B \mathrm{e}^{-\beta t}+C$

$$
(\beta>\alpha>0 ; A B<0 ; c<0 ; \beta|B|>\alpha|A|) .
$$

Le pompage est appliqué soudainement à l'instant $t=0$.

Le modèle précédent a reçu diverses extensions :

- représentation de l'état macroscopique par la matrice densité [12] :

- évolution des populations lorsque la multiplicité fondamentale est d'ordre 4 [5] ;
- étude du fonctionnement en oscillateur [12] et prévision de "spikes» analogues à ceux du laser déclenché.

2. Principe du maser pompé par dichroïsme. - Le pompage s'effectue dans une bande large (avec descente rapide au niveau 3) et affecte les deux populations $n_{1}$ et $n_{2}$ avec les probabilités $W_{\mathrm{p} 1}$ et $W_{\mathrm{p} 2}$. Le dichroïsme se traduit par $W_{\mathrm{p} 1} \neq W_{\mathrm{p} 2}$. Si, de plus, $W_{\mathrm{p} 1}>W_{\mathrm{p} 2}$, il peut y avoir inversion pour les niveaux 1 et 2 . Le pompage doit aussi vérifier : $W_{\mathrm{p} 1}$ et $W_{\mathrm{p} 2} \ll w_{31}$ et $w_{32}$, pour ne pas dépeupler la multiplicité 1,2 fondamentale. Avec cette condition et les hypothèses $a, b, d, e, f \mathrm{du} \S 1$, on obtient, en régime stationnaire [14]

$$
\left(\frac{n_{2}-n_{1}}{N}\right)_{\mathrm{st}}=\frac{\eta P-\frac{\hbar \omega_{\mathrm{s}}}{k T} r}{P+2 r},
$$

où

$$
\begin{gathered}
r=\frac{w_{12}+w_{21}}{2}, P=\frac{W_{\mathrm{p} 1}+W_{\mathrm{p} 2}}{2}, \\
\eta=\frac{W_{\mathrm{p} 1}-W_{\mathrm{p} 2}}{W_{\mathrm{p} 1}+W_{\mathrm{p} 2}} \text { et } N=n_{1}+n_{2}+n_{3} .
\end{gathered}
$$

La condition de fonctionnement optimal est :

$$
P \gg 2 r
$$

et, dans ce cas, $\left(n_{2}-n_{1}\right)$ se réduit à $\eta N$.

3. Bruit. - La superposition des différentes causes de bruit se traite, comme pour les autres masers, au moyen de circuits équivalents [18]. Nous n'étudions ici que le bruit produit, dans le cristal même, par émission spontanée. Pound a montré [19-20] que la température $T_{\mathrm{m}}$ caractérisant ce bruit égale la valeur absolue de la température de spin réalisée. Dans la mesure où le pompage optique produira une inversion notable à température relativement élevée, la température de bruit obtenue sera très inférieure à la température extérieure. Au contraire, dans le maser à pompage hyperfréquence, les populations d'équilibre se traduisent par une température de spin, dont la valeur absolue est toujours voisine de la température extérieure [17].

4. Réalisations. - (Voir tableau). Les masers à pompage sélectif utilisent le rubis, dont on connaît avec précision les caractéristiques: niveaux fondamentaux [21], [17] et excités [22], relaxation [23-24] et durées de fluorescence [25]. Le fonctionnement est impulsionnel, du fait de la forme du pompage et aussi parce que, dans la plupart des cas,

$$
\omega_{\mathrm{s}}>\omega_{0}\left(\omega_{0} / 2 \pi \simeq 10 \mathrm{GHz} \text { à } 77^{\circ} \mathrm{K}\right) \text {. }
$$

Un problème délicat est celui de l'accord de fréquence entre l'émission laser et la transition de pompage ; la fréquence laser est décalée par rapport à la fluorescence [26] et elle varie au cours de l'impulsion [27]. 
Une tentative [15] pour obtenir l'inversion de populations de niveaux fondamentaux dans un cristal laser en action $\left(\mathrm{Al}_{2} \mathrm{O}_{3}: \mathrm{V}^{3+}\right)$ a été infructueuse.

Les masers pompés par dichroïsme fonctionnent en continu avec une simple lampe à arc. La condition (3) impose une température basse (ici hélium liquide); pour une puissance de pompage raisonnable (un watt absorbé environ), le temps de relaxation spin-réseau $r^{-1}$ doit, en effet, être au moins de l'ordre de la seconde.

Conclusions. - Les masers pompés par lasers sont peu pratiques comme préamplificateurs de réception en raison de leur fonctionnement impulsionnel. Cependant, leur mise au point a suscité de nouvelles recherches intéressantes [28], [29]. D'autre part, leurs possibilités de fonctionnement à plus haute fréquence (jusqu'à $270 \mathrm{GHz}$ ) ont été examinées [30].

Les masers pompés par dichroïsme semblent plus aptes à application technique. Leur domaine de fonctionnement actuel $\left(4{ }^{\circ} \mathrm{K}, 20 \mathrm{GHz}\right)$ est équivalent à celui des masers pompés par hyperfréquences mais ce domaine pourrait être étendu (sans limitation due à la pompe) par l'usage de bobines supraconductrices.

\section{TABLEAU}

Référence
$[8-[11]$
$[9]$
$[10]$
$[12]$
$[13]$
$[14]$

Référence

[8], [11]

9]

$[12]$

$[14]$

$\quad \stackrel{\text { Pompe }}{-}$
laser rubis
laser rubis
laser rubis
laser rubis
arc $\mathrm{Hg} 500 \mathrm{~W}$
arc $\mathrm{Hg} 500 \mathrm{~W}$

Cristal maser
rubis
rubis
rubis
rubis
$\mathrm{CaF}_{2}: \mathrm{Tm}^{++}$
$\mathrm{SrF}_{2}: \mathrm{Tm}^{++}$

$\begin{array}{cc}\begin{array}{c}\text { Fonction- } \\ \text { nement }\end{array} & \begin{array}{c}\text { Température } \\ (\mathrm{O} \mathrm{K})\end{array} \\ \text { impulsions } & 4,2 \\ \text { impulsions } & 77 \\ \text { impulsions } & 78-82 \\ \text { impulsions } & 77 \\ \text { continu } & 1,4-4 \\ \text { continu } & 1,4\end{array}$

\section{Bibliographie}

[1] Series (G. W.), TAYlor (M. J.), J. Phys. Radium, 1958, 19, 901.

[2] Theissing (H. H.), Caplan (P. J.), Dieter (F. A.), Rabbiner (N.), Phys. Rev. Letters, 1959, 3, 460.

[3] Brossel (J.), Quantum Electronics, Columbia Un. Press, 1960, 81.

[4] Szabo (A.), Bull. Rad. and Electr. Engrg. Div., Natl Research Council of Canada, 1951, 11, 52.

[5] Ready (J. F.), Chen (D.), P. I. R. E., 1962, 50, 329.

[6] Hsu (H.), Tittel (F. K.), P. I. R. E., 1963, 51, 185.

[7] Karlov (N. V.), Margerie (J.), Merle D’Aubigné (Y.), J. Physique, 1963, 24, 717.

[8] Devor (D. P.), D’Haenens (I. J.), Asawa (C. K.), Phys. Rev. Letters, 1962, 8, 432.

[9] Zverev (G. M.), Prokhorov (A. M.), Shevchenko (A. K.), Zh. Eksper. Teor. Fiz., 1963, 44, 1415. Traduction Soviet Physics, J.E.T.P., 1963, 17, 952.

[10] Szabo (A.), P. I. R. E., 1963, 51, 1037.

[11] Devor (D. P.), D'Haenens (I. J.), Asawa (C. K.), $3^{\text {e }}$ Conférence d'Electronique Quantique, Paris, 1963, 931.

[12] Saito (F.), Jap. Journ. Appl. Phys., 1967, 6, 89.

[13] Sabisky (E. S.), Anderson (C. H.), Appl. Phys. Lett., 1966, 8, 298.

[14] SAbisky (E. S.) et Anderson (C. H.), 1. E. E. E., J. Quantum Electronics, 1967, QE 3, 287.
[15] Clark (J. C.), Grow (R. W.), 7th Inter. Conf. on microwave and opt. gén. and amplif. - Hambourg (16-20 sept. 1968).

[16] Bloembergen (N.), Phys. Rev., 1956, 104, 324.

[17] Weber (J.), Rev. Mod. Phys., 1959, 31, 681.

[18] Siegman (A. E.), P. I. R. E., 1957, 45, 1737.

[19] Pound (R. V.), Ann. Physique, 1957, 1, 24.

[20] Bertein (F.), Bases de l'Electronique quantique (Eyrolles, Paris, 1969, t. II, ch. X.

[21] Chang (W. S.), Siegman (A. E.), Stanford Electronic Laboratory, Technical Report, $\mathrm{n}^{\circ}$ 156-2, Stanford University.

[22] Geshwind (S.), Collins (R. J.), Schawlow (A. L.), Phys. Rev. Letters, 1959, 3, 548.

[23] Gill (J. C.), Quantum Electronics, C. H. Townes, Edit., Columbia University Press, New York, 1960.

[24] Gill (J. C.), Proc. Phys. Soc., 1962, 79, 58.

[25] Varsanyi (F.), Wood (D. L.), Schawlow (A. L.), Phys. Rev. Letters, 1959, 3, 545.

[26] D'Haenens (I. J.), Asawa (C. K.), J. Appl. Phys., 1962, 33, 3201.

[27] Hugues (T. P.), Nature, 1962, 195, 325.

[28] Igarashi (T.), Szabo (A.), I. E. E. E., Journ. Quant. Electr., 1967, QE 3, 143.

[29] Szabo (A.), J. Appl. Phys., 1968, 39, 5425.

[30] Devor (D. P.), 1.E.E.E., Trans. Micr. Theor. and Techn., M. T. T., 1963, 11, 251. 